\title{
The FARCOS project. First characterization of detectors and front-end electronics of FARCOS array
}

\section{L.Quattrocchi ${ }^{4,5 *}$}

L.Acosta $^{2,8}$, F.Amorini ${ }^{2}$, A.Anzalone ${ }^{2}$, L.Auditore ${ }^{4,5}$, G.Cardella ${ }^{1}$, E.De Filippo ${ }^{1}$, L.Francalanza $^{2,3}$, R.Giani ${ }^{2,3}$, G.Lanzalone, ${ }^{2,7}$, I.Lombardo ${ }^{6}$, T.Minniti, ${ }^{4,5}$, E. Morgana ${ }^{5}$, S. Norella $^{5}$, A.Pagano ${ }^{1}$, E.V.Pagano ${ }^{2,3}$, M.Papa ${ }^{1}$, S.Pirrone ${ }^{1}$, G.Politi ${ }^{1,3}$, F.Rizzo $^{2,3}$, P.Russotto ${ }^{1}$, A.Trifirò $^{4,5}$, M.Trimarchì ${ }^{4,5}$, G.Verde $^{1}$

1- INFN Sezione di Catania, Catania, Italy

2- INFN LNS, Catania, Italy

3- Dipartimento di Fisica e Astronomia Università di Catania, Catania, Italy

4- INFN Gruppo Collegato di Messina, Messina, Italy

5- Dipartimento di Fisica e Scienze della Terra Università Messina, Messina, Italy

6- INFN-Sezione di Napoli and Dipartimento di fisica Università di Napoli Federico II, Napoli, Italy

7- Università Kore Enna, Enna, Italy

8- Departamento de Física Aplicada, Universidad de Huelva, Huelva, Spain

E-mail: Iquattrocchi@unime.it

The construction of a new array to study femtoscopy and multi-particle correlations in heavy-ion collisions at intermediate energies $(\mathrm{E}=20-1000 \mathrm{AMeV})$ has been started at the INFN of Catania (Sezione and LNS). The project, named FARCOS (Femtoscope ARray for COrrelations and Spectroscopy) is aimed to the development of a detector system with high pixelation capabilities, in order to perform high precision measurements of two- and multi-particle correlations. The detector will address topics related to the study of dynamics and the equation of state of asymmetric nuclear matter, as well as spectroscopy with both stable and exotic beams. We present first detection simulations for FARCOS and first experimental results related to the commissioning of detectors and of front end electronics.

52 International Winter Meeting on Nuclear Physics

January 27 - 312014

Bormio, Italy

Speaker. 


\section{Introduction}

Energetic heavy-ion collisions have been extensively studied to extract information about the properties of nuclear matter under extreme conditions of density and temperature. Measuring the properties of the produced nuclear systems represents the only terrestrial means to access the equation of state of nuclear matter. In order to extract such nuclear matter properties, a clear understanding of the complex dynamics of heavy-ion collisions is required. Such challenge can be accomplished by using two- and multi-particle correlations. The investigation of two-particle correlation functions at small relative momenta, can provide important information about the space-time characteristics and underlying dynamics of particle emitting sources [1]. These studies have also shown that a large variety of isotopes is produced during the dynamical evolution of the reaction. Some of these isotopes, lying far from the stability line, live temporarily and decay by particles emission. Their unbounded states can then be isolated and studied by means of correlation techniques. As an example, p- ${ }^{7} \mathrm{Be}$ correlation function have been measured in central collision $\mathrm{Xe}+\mathrm{Au}$ at $\mathrm{E}=50 \mathrm{AMeV}$ [2] to probe the spin of the astrophysical important state around $0.774 \mathrm{MeV}$ in ${ }^{8} \mathrm{~B}$.

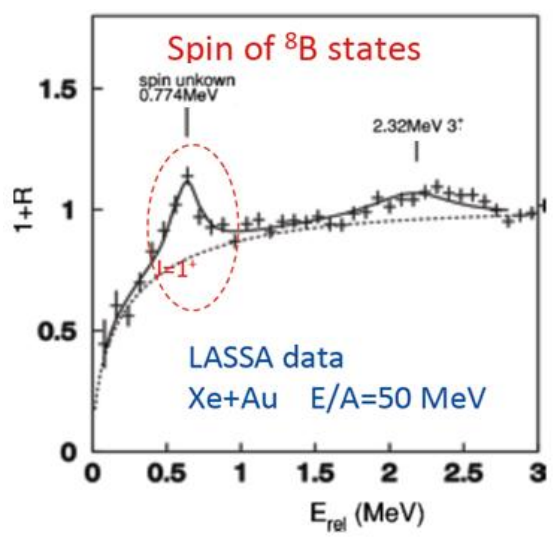

Figure. 1- ${ }^{7} \mathrm{Be}$-proton correlation function measured in $\mathrm{Xe}+\mathrm{Au}$ collisions a $\mathrm{E}=50 \mathrm{AMeV}$. See reference [2] for details.

In this respect, a collision between two heavy-ions can be viewed not only as a tool to study nuclear dynamics but also as a laboratory to produce several species in one single experiment and study their spectroscopic properties. To reach highly precise experimental results, needed to study such interactions, detectors capable of detecting all reaction products and measure their reciprocal correlations are mandatory. Different observables need to be measured over a large solid angle (ensuring a good characterization of the collision event) with high energy and angular resolution (to register the kinetic energy and to obtain the momentum vectors and their correlations). The FARCOS (Femtoscope ARray for COrrelations and Spectroscopy) project is aimed to the development of a detection system with high pixelation 
capabilities, to perform precision measurements of particle correlations for nuclear dynamics and spectroscopy with stable and radioactive beams.

\section{FARCOS array}

FARCOS has been conceived as a compact high resolution array; the basic telescope consists of two double-sided silicon strip detectors (DSSSD) 300 and $1500 \mu \mathrm{m}$ thick, as first and second stage respectively, followed by $4 \mathrm{CsI}(\mathrm{Tl})$ crystals arranged in square configuration $2 \times 2$, as a third stage. The total detection area of silicon detectors is $64 \times 64 \mathrm{~mm}^{2}$, adapted to cover the total area of the $4 \mathrm{CsI}(\mathrm{Tl})$ crystals placed behind. The scheme of the different stages of one FARCOS cluster is shown in fig. 2.

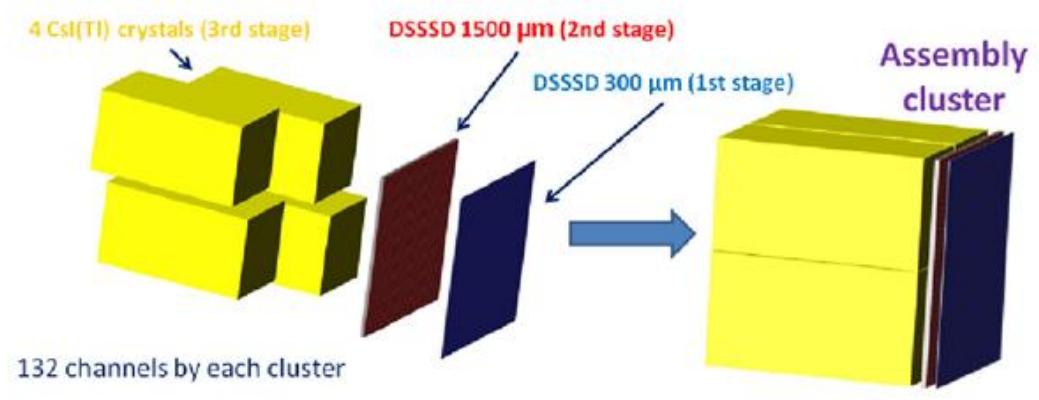

Figure. 2- Schematic representation of a FARCOS telescope.

Each DSSSD features 32 horizontal and 32 vertical strips, providing 1024 equivalent pixels of $2 \times 2 \mathrm{~mm}^{2}$. This segmentation allows to reconstruct with high accuracy the impact position of detected particles, hence their emission angles with high resolution. According to the topics exposed in the previous section, the FARCOS project presents peculiar features. In addition to high energy and angular resolution, FARCOS will have a wide dynamic range going from $\mathrm{MeV}$ to $\mathrm{GeV}$; the high stopping power of thick $\mathrm{CsI}(\mathrm{Tl})$ allows indeed to stop high energy light particles, while low thresholds for particle identification will be attained with pulse shape techniques[5]. Silicon nTD solutions are also under consideration to improve pulse-shape capabilities, as observed in some experiments[3]. Furthermore, the flexible geometry allows us to arrange FARCOS telescopes to cope with the physical case under investigation; it has been conceived as a portable device, so it can be coupled with other existing detectors. It will be interesting to use the FARCOS array in coincidence with large $4 \pi$ arrays (such as CHIMERA or INDRA), to have a good characterization of the collision events. Coupling to magnetic spectrometers and neutron detectors is also under study, to extend the physics targets of project to spectroscopy studies conducted with stable and exotic beams. 


\section{Simulation results}

In order to understand the influence of angular resolution on reconstruction of physical observables of interest, hence on correlation functions, preliminary simulations were performed. It was chosen to simulate the decay of ${ }^{8} \mathrm{~B}$ in ${ }^{7} \mathrm{Be}$ and a proton and it was assumed that ${ }^{8} \mathrm{~B}$ is evaporated, with a Maxwell velocity distribution, by a source moving in the laboratory reference system; a temperature of $7 \mathrm{MeV}$ was chosen for the source. Simulated data were sent through a filter that can reproduce all geometrical configuration of FARCOS array; the software takes into account all physical and geometrical features of the apparatus and it is also very useful in order to compare theoretical predictions with experimental observations. To show the effects of improvement of angular resolution, the results obtained using two different setups were compared. The first consists in only one telescope, the last by nine different telescopes arranged in $3 \times 3$ configuration. The two setups cover the same angular region but with different angular resolution, as evidenced by the plot in Fig. 3: in the first case the average polar resolution is about $0.8^{\circ}$, while in the second one is $0.15^{\circ}$.
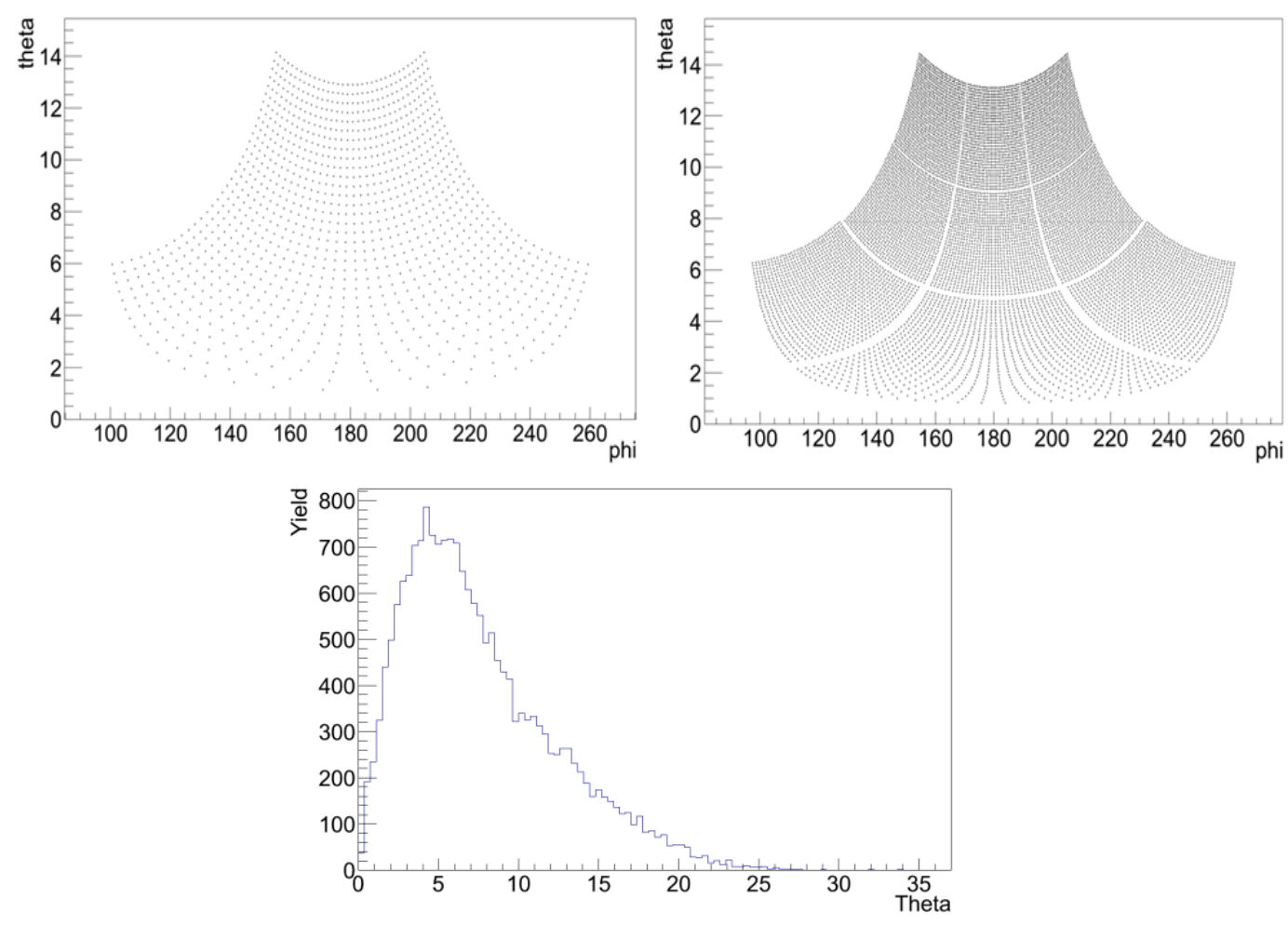

Figure 3. - Upper panel: angular resolution obtained with one (left panel) and nine (right panel) FARCOS telescopes covering the same angular region; the points in this plot represent the spherical coordinates of center of each pixel. Lower panel: polar distribution of proton and ${ }^{7} \mathrm{Be}$ produced in the simulated decay of ${ }^{8} \mathrm{~B}$. 
The scatter plots shown in Fig. 4 report the ratio between number of pairs detected by simulated configurations and the total number produced as a function of relative energy $\mathrm{E}_{\text {rel }}$.
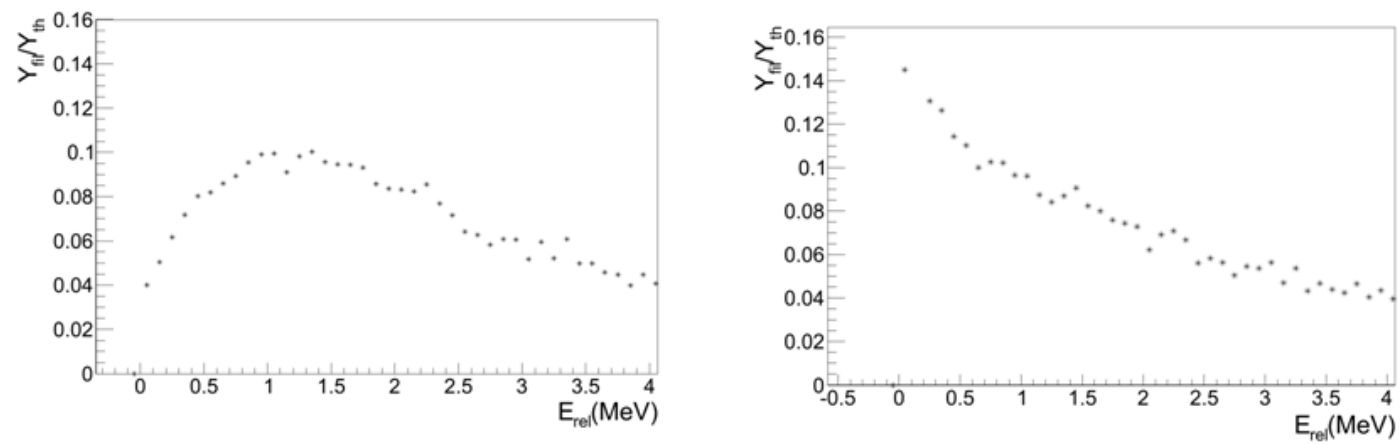

Figure 4. - Scatter plots of ratio of number of particles pairs and number of produced pairs vs. the relative energy $\mathrm{E}_{\text {rel }}$ (left panel: one telescope; right panel: nine telescopes).

For the second setup an enhancement of efficiency is observed. In particular in the regions of interest of $\mathrm{p}-{ }^{7} \mathrm{Be}$ correlation function, around the first excited level of ${ }^{8} \mathrm{~B}$ at $0.774 \mathrm{MeV}$, it is possible to obtain an improvement of efficiency by about a factor of 3 by using the setup with nine telescopes. A similar improvement is observed for relative energy reconstruction. The plots in fig. 5 report the deviation between the simulated relative energy and the same observable filtered by the chosen apparatus:

$$
\delta E_{r e l}=\frac{E_{f i l}-E_{t h}}{E_{t h}}
$$

for small values of relative energy the error in reconstruction of the same observable increases: for the first setup these errors exceed $20 \%$, while for the second one they are of few percentage points.
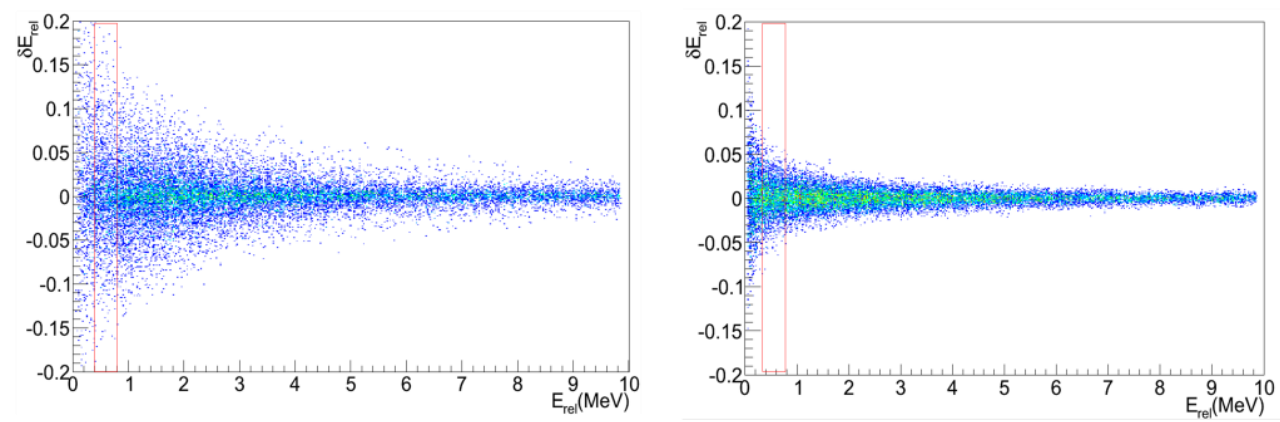

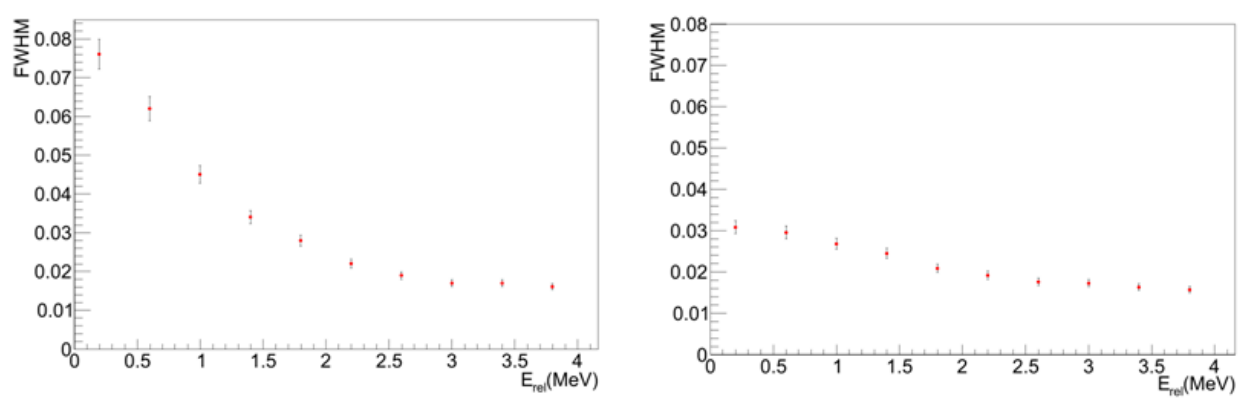

Figure 5. - Upper panel: deviation between the simulated relative energy and same observable filtered by chosen apparatus (left panel: only one telescope; right panel: nine different telescopes). Lower panel: FWHM of $\delta E_{\text {rel }}$ distributions as function of relative energy for both setups.

In the lower panel of fig. 5 the FWHM of $\delta E_{\text {rel }}$ distribution as a function of relative energy for both setups are reported in order to show the enhancement of energy resolution when nine telescopes are used. For $\mathrm{E}_{\text {rel }}$ values between 0.4 and $0.8 \mathrm{MeV}$, in the second setup the energy resolution increases by a factor around 3 with respect to the first one. Hence, with a suitable setup it is possible to achieve efficiency and resolution required for a proper reconstruction of correlation functions.

\section{Experimental results}

A good reconstruction of the relative energy depends on both angular and energy resolution of the single telescope. High energy resolution is also required in spectroscopy applications in which it is important to separate energy states very close together. For this reason several tests were conducted to estimate the performance of each identification stage of FARCOS telescopes, with special attention to energy resolution. Concerning the test on DSSSD, possible charge sharing phenomena were investigated with a mixed alpha source. Each strip is separated by an insulating material of $\mathrm{SiO}_{2}$; when a particle cross this region can release part of its energy in a strip and part in its neighboring one.

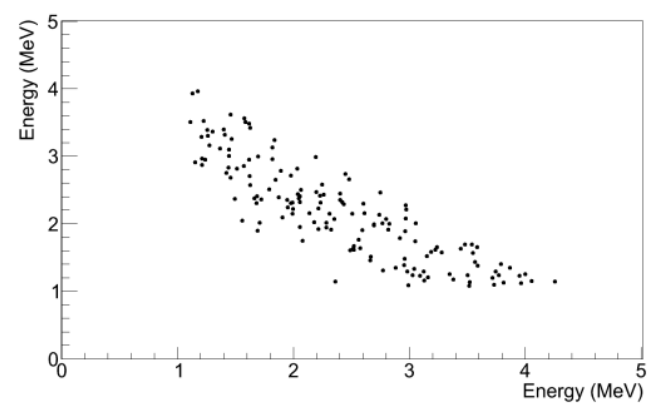

Figure 6. - Energy released in a strip as function of energy released in its adjacent one with the condition that they are hit in coincidence. 
The plot in fig. 6 reports the energy read by one strip as a function of that read by the adjacent one under the condition that they are hit in coincidence; the points in the plot are approximately located along three parallel lines thus showing the presence of such sharing phenomena. Indeed the total energy released in two adjacent strips should be constant and equal to the energy of the used source.
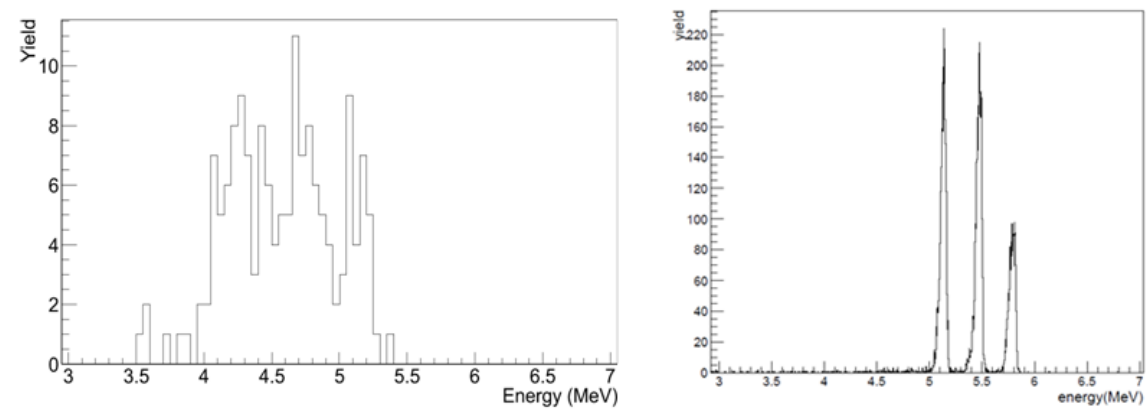

Figure 7. - Left panel: energy spectrum obtained by adding the energies released in two adjacent strips (charge sharing events). Right panel: alpha spectrum acquired with a single strip.

In the left panel of fig. 7 the spectrum obtained by adding the energies released in two adjacent strips (charge sharing event) is reported; the peaks are shifted toward lower energy probably because the particle could release part of its energy in the interstrip zone in a random way. This means that it is impossible to recover these events. Nevertheless they can't effect significantly the statistic and resolution of the measurements because they represent only the $0.1 \%$ of total ones. From these tests on DSSSD, good resolution have emerged, needed for to our studies; with a mixed alpha source (about $5 \mathrm{MeV}$ ) the $300 \mu \mathrm{m}$ silicon detectors show a resolution of about $45 \mathrm{KeV}$ as shown in right panel of fig.7. The overall energy resolution of FARCOS telescopes depends mainly on the quality of the CsI(Tl) crystals. Several factors such as dopant concentration gradient, imperfections in crystal wrapping and/or in photodiode coupling can affect the energy resolution of this scintillation detector. In particular, the aspect that deserves special attention is a possible position dependence of light output of these crystals. For this reason, special tests have been carried out using firstly a collimated monochromatic alpha source, to scan the surface of the crystal and, after different particles beams to investigate a possible non-uniformity in depth, searching for noticeable differences in the output signals. The dependence on the position of the signal produced has been tested by means of an ${ }^{241} \mathrm{Am} \alpha$ source. A special apparatus has been setup allowing to move the $\alpha$-CsI(Tl) system in perpendicular directions inside the vacuum chamber. The system was automatized in such a way that an energy spectrum was obtained for points separated by spacing of $2.7 \mathrm{~mm}$ on a 3 by 3 Cartesian grid on the front surface of the tested crystals. Using the information from the linear drivers used to move the source, discrete grids in the $x-y$ coordinate plane are mapped on the 
crystal surface. At the coordinate $(x, y)=(i, j)$, the non-uniformity of light output is expressed as $S_{i j}=\left(L_{i j}-\langle L\rangle\right) /\langle L\rangle[6] ; L_{i j}$ represents the centroid of energy peak at $(\mathrm{i}, \mathrm{j})$ position, while $\langle L\rangle$ represents the average over the entire crystal surface. Fig. 8 shows the light response for two different crystals: in the left panel the non-uniformities exceed $4 \%$ while in the right one a very good uniformity (better than 1\%) is observed.
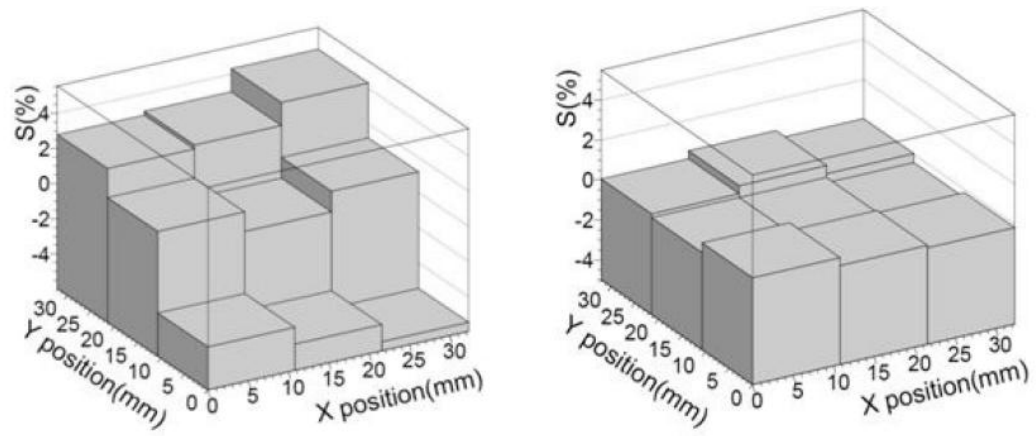

Figure 8. - Characterization of light response of two tested CsI(Tl) crystals obtained with alpha source [7].

Based on these results, it is important to study the position dependence of light response with higher energy particles. While $\alpha$ source tests involve a region of the crystal that extend up to only a few microns beyond the entrance window, measurements of light output performed with beam at higher energy allow one to explore the quality and uniformity in crystal response at different depths inside. To this aim an analysis was performed with data collected during a test made at LNS of Catania during last months. In particular data related to the reaction $\alpha$ $+{ }^{208} \mathrm{~Pb} @ 62 \mathrm{AMeV}$ were used.
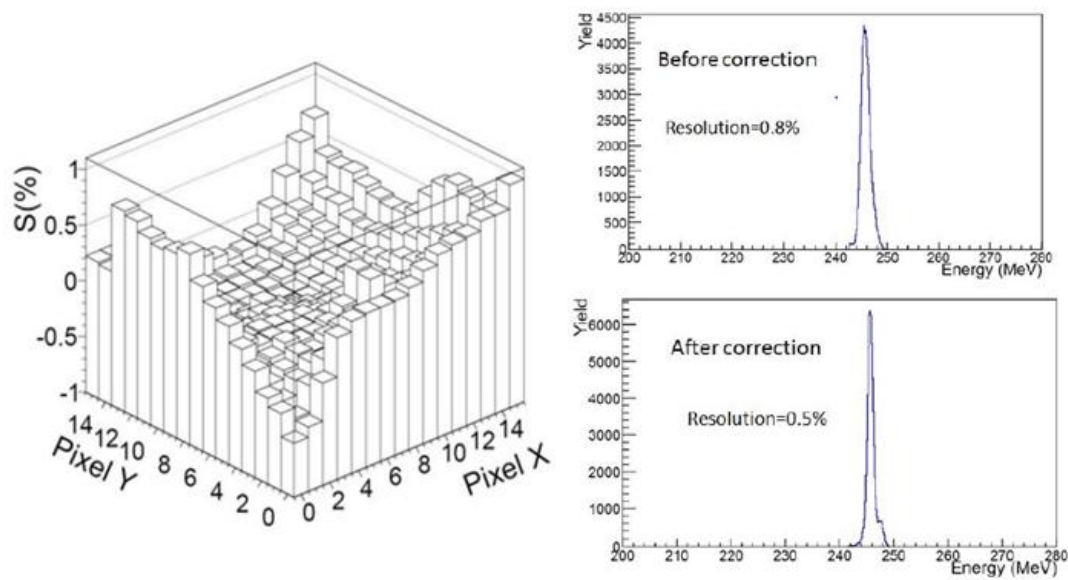

Figure 9. - Left panel: Characterization of light response for one of the tested CsI(Tl) detector using an alpha beam at $62 \mathrm{AMeV}$ scattered by ${ }^{208} \mathrm{~Pb}$. Right panel: total energy spectrum acquired with a CsI(Tl) detector before and after correcting for the light response of each pixel with respect to the average response over the entire crystal. 
To search and identify any position dependence in the crystal light output, the position information is given by the DSSSD placed in front of the CsI(Tl) crystals. The strips provide tracking information by dividing the active area of silicon detectors into 1024 pixels, hence into 256 pixels for each crystal. Fig. 9 (left panel) reports light response of one tested crystal using $62 \mathrm{AMeV} \alpha$ beam, scattered by ${ }^{208} \mathrm{~Pb}$. For all the analyzed crystals the non-uniformity doesn't exceed $1 \%$, then it is possible to distinguish energy variations of $2 \%$. The knowledge of the impact position (provided by the front DSSSD) will allow us to correct for light response nonuniformities, thus improving the final energy resolution. As shown in fig. 10 (right panel) energy resolution of $\mathrm{CsI}(\mathrm{Tl})$ can be improved from $0.8 \%$ to $0.5 \%$ after taking into account the light response of each pixel with respect to the average response of the crystal.

In parallel to these tests a new front-end electronics optimized for the FARCOS requirement is being developed. Of course one of the main problems related to this array is the control and processing of signals produced by each channel (132 for each telescope). Some tests are in progress to evaluate the possibility to using a GET electronics (General electronics for TPCs) to process the signals produced by FARCOS detectors. GET is an ASIC electronics with digitization system that allow to have a great number of channel in a very compact modules.

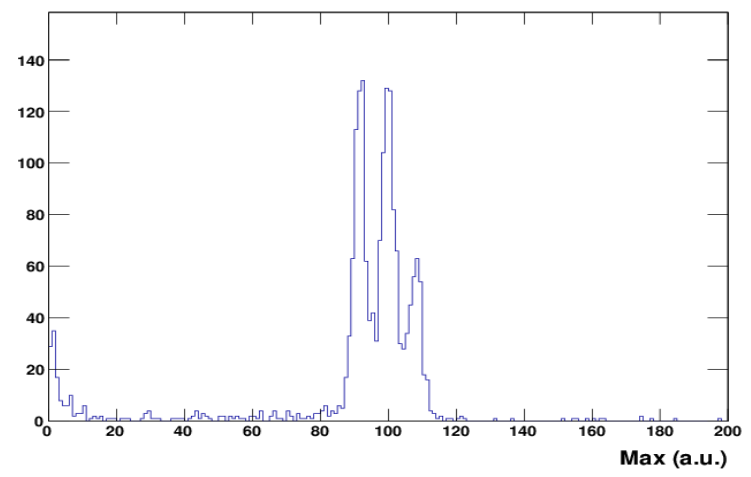

Figure 10- Spectrum of a mixed alpha source obtained with a CsI(Tl) crystal of FACOS telescope using a channel of GET electronics.

The plot in fig. 10 reports the spectrum of a mixed alpha source obtained with a $\mathrm{CsI}(\mathrm{Tl})$ detector, using a channel of GET electronics[8]. A energy resolution of $180 \mathrm{KeV}$ was observed, and it is comparable with what obtained with traditional electronics.

\section{Conclusions}

The FARCOS array under construction at the INFN Sezione di Catania and LNS (Laboratori Nazionali del Sud), will allow to perform a good measurement of correlation functions for nuclear dynamics and spectroscopy. In this paper results of simulations and test made on FARCOS detectors were discussed. The simulations emphasized the key role of angular resolution in the reconstruction of important physical observables used to build the 
correlation functions. The tests made on DSSSD showed a very good resolution, needed for the study planned in the project; while possible non-uniformities in light response of $\mathrm{CsI}(\mathrm{Tl})$ crystals were estimated. By studying these non-uniformities in light response it is possible to apply a corrections that can improve energy resolution of the crystals. In parallel to these tests, a new front-end electronics, optimized for the FARCOS requirement, is being developed. In particular, some tests to evaluate the possibility of using GET electronics to process the signals produced by FARCOS telescopes are in progress. Indeed ASICS electronics with a digitalization system allow to obtain more compact modules and a better processing of signals produced by individual channels.

\section{References}

[1] Verde et al., Eur. Phys. J. A30 (2006) 81.

[2] W.P. Tan et al., Physical Review c69, 061304(R) 2004

[3] L. Mutterer et al., Nuclear Instrument and Methods in Physics Research A 608 (2009) 275

[4] L. Bardelli et al., Instrument and Methods in Physics Research A605 (2009) 353.

[5] A. Alderighi et al., IEEE Trans. On Nucl. Sci. 52, (2005) 1624

[6] M-J. Van Goethem et al., Nuclear Instrument and Methods in Physics Research A526 (2004) $455-476$

[7] T. Minniti, Study of Correlation Functions in Heavy-Ion Collision, Ph.D. Thesis, Messina University 2013.

[8] T. Minniti, FARCOS, GASPARD-HYDE-TRACE Workshop 2014. 